\title{
The comparison of effectiveness of therapy with ustekinumab and etanercept in psoriatic patients during 48 weeks' observation
}

\author{
Dominika Wcisło-Dziadecka1', Beniamin Grabarek ${ }^{2,3,4}$, Joanna Gola ${ }^{4}$, Andrzej Plewka ${ }^{5}$
}

1Department of Cosmetology, Faculty of Pharmaceutical Sciences, Medical University of Silesia, Katowice, Poland 2Department of Histology, Cytophysiology and Embryology, Faculty of Medicine in Zabrze, University of Technology in Katowice, Poland ${ }^{3}$ Department of Nursing and Maternity, High School of Strategic Planning, Dąbrowa Górnicza, Poland ${ }^{4}$ Department of Molecular Biology, Faculty of Pharmaceutical Sciences, Medical University of Silesia, Katowice, Poland ${ }^{5}$ Institute of Health Sciences, University of Opole, Opole, Poland

Adv Dermatol Allergol 2021; XXXVIII (1): 173-175 DOI: https://doi.org/10.5114/ada.2021.104295

Inhibitors of the tumour necrosis factor (TNF) - adalimumab, etanercept and 12/23 (IL-12/23) interleukin inhibitor - ustekinumab are used in the therapy of psoriasis vulgaris and psoriatic arthritis. These inhibitors suppress signal pathways activated by the cytokines, for which the said medicines were designed [1].

Etanercept is an example of a fusion protein which bonds to the soluble form of TNF. In turn, ustekinumab shows ability to bind to subunit p40 (IL12B), common for IL-12 and IL-23. The efficacy of therapies with the use of the anti-cytokine medicines can be achieved following a 12-week long pharmacotherapy [2]. Psoriasis Area and Severity Index (PASI), Dermatology Life Quality Index (DLQI) and Body Surface Area (BSA) scales are used to evaluate the efficacy. Application of the three indexes, which enable to assess, either objectively or subjectively, the intensity of symptoms and therapy efficacy, makes it possible to eliminate the possible interpretation errors of each scale separately. Moreover, thanks to the three said scales it is possible to get a holistic view of the patients' disease and monitor how the applied therapy affects the quality of their lives [3].

The aim of our study was to compare the efficacy of therapies with the use of etanercept and ustekinumab during a 48-week long observation of psoriatic patients, on the basis of the recommended scales of disease advancement. We also made an attempt to determine whether during the molecularly targeted therapy, a loss of adequate response to treatment (drug resistance) can occur, how fast it can be detected and which of the indexes may describe the case the most accurately.
The sample group was composed of 10 persons, including 7 people with diagnosed psoriasis vulgaris treated with ustekinumab and 3 people with diagnosed psoriatic arthritis treated with etanercept, who had the therapeutic effects analysed 5 times during the whole observation period.

The efficacy was assessed with the use of PASI, BSA and DLQI scales in patients treated with ustekinumab on week 0-4-16-28-40, which resulted from the exact dosage regime. In turn, in case of patients treated with etanercept, the observation was made basing on the PASI, BSA and DLQI values every 12 weeks (week 0-12-24-36-48).

The statistical analysis $(p<0.05)$ was performed with the use of Statistica 12 (Krakow, Poland). The values of PASI, BSA and DLQI indexes in a given week was described with the use of a median (Me), the lower quartile (Q1), and the upper quartile (Q2). In order to determine the changes in the values of PASI, BSA and DLQI indexes in the groups of patients with psoriasis vulgaris and patients with psoriatic arthritis, the ANOVA Friedman, and then the post-hoc ANOVA Friedman tests were performed.

When analysing the changes in values of the individual scales in the group of patients treated with ustekinumab, one may observe a decrease in the values of the said parameters between the period of therapy commencement and observation completion in week 40 (PASI -10.5; BSA -4.00; DLQI -32.7). The statistical analysis showed two statistically significant differences in the values of the individual indexes during ustekinumab therapy, whereas the first important difference was observed

Address for correspondence: Dominika Wcisło-Dziadecka MD, PhD, Department of Cosmetology, Faculty of Pharmaceutical Sciences, Medical University of Silesia, 3 Kasztanowa St, 41-200 Sosnowiec, Poland, phone/fax: +48 3225 91 580/581, +48 32 256-11-82, e-mail: ddziadecka@sum.edu.pl Received: 5.02.2019, accepted: 7.07.2019. 
after 16 weeks of the pharmacotherapy. As far as PASI is concerned, apart from week 16 , its value systematically decreased. The BSA value remained relatively stable during 52 weeks of therapy. In turn, increased DLQI value was observed only in week 28 , with a drop in all other periods (Table 1).

Whereas, among the patients treated with etanercept, the differences in the values of the disease progress clinical parameters between week 0 and week 48 were lower than those noted for ustekinumab (PASI -2.00; BSA +2.00; DLQI -2.00). The opposite direction was observed for PASI and BSA, i.e. an increase in one of the said parameters involved a concurrent decrease in the other and vice versa. In turn, the value of DLQI decreased in weeks 16 and 28 , remaining at the comparable level during the other periods or as before therapy commencement. The statistical analysis did not show any statistically significant differences in PASI, BSA and DLQI values during the 48-week long period of etanercept therapy (Table 1).

In this study, we analyse changes in the values of PASI, BSA and DLQI parameters during treatment with etanercept and ustekinumab during 48 weeks of observation. Basing on the differences in the presented scales, we evaluated the efficacy of the therapies with the use of both medicines and made an attempt to determine which anti-cytokine therapy seems to be more effec-

Table 1. PASI, BSA and DLQI values in patients with psoriasis vulgaris and psoriatic arthritis treated with ustekinumab and etanercept ( $p<0.05$; NS - statistically insignificant differences)

\begin{tabular}{|c|c|c|c|c|c|c|}
\hline Group & Scale & Time of therapy [weeks] & Median & Bottom quartile & Upper quartile & $\begin{array}{c}\text { ANOVA Friedman } \\
p<0.05\end{array}$ \\
\hline \multirow[t]{15}{*}{ Ustekinumab } & \multirow[t]{5}{*}{ PASI } & 0 & 25.90 & 22.70 & 29.10 & \multirow{5}{*}{$\begin{array}{c}0 \text { vs. } 16 p=0.01 \\
0 \text { vs. } 28 p=0.00086 \\
0 \text { vs. } 40 p=0.000253\end{array}$} \\
\hline & & 4 & 21.00 & 15.00 & 23.00 & \\
\hline & & 16 & 59.00 & 41.00 & 63.00 & \\
\hline & & 28 & 15.40 & 9.30 & 17.10 & \\
\hline & & 40 & 9.00 & 8.00 & 15.00 & \\
\hline & \multirow[t]{5}{*}{ BSA } & 0 & 9.00 & 0.00 & 10.00 & \multirow{5}{*}{$\begin{array}{l}0 \text { vs. } 16 p=0.048496 \\
0 \text { vs. } 28 p=0.007312 \\
0 \text { vs. } 40 p=0.000225\end{array}$} \\
\hline & & 4 & 9.00 & 5.00 & 14.00 & \\
\hline & & 16 & 2.40 & 1.60 & 5.60 & \\
\hline & & 28 & 6.00 & 1.00 & 8.00 & \\
\hline & & 40 & 5.00 & 3.50 & 9.00 & \\
\hline & \multirow[t]{5}{*}{ DLQI } & 0 & 35.00 & 26.00 & 40.00 & \multirow{5}{*}{$\begin{array}{l}0 \text { vs. } 16 p=0.009690 \\
0 \text { vs. } 28 p=0.0011910 \\
0 \text { vs. } 40 p=0.000965\end{array}$} \\
\hline & & 4 & 6.40 & 2.50 & 11.00 & \\
\hline & & 16 & 6.00 & 1.00 & 10.00 & \\
\hline & & 28 & 23.00 & 5.00 & 29.00 & \\
\hline & & 40 & 3.20 & 2.10 & 6.70 & \\
\hline \multirow{15}{*}{ Etanercept } & \multirow{5}{*}{ PASI } & 0 & 10.40 & 2.00 & 18.00 & \multirow{5}{*}{ NS } \\
\hline & & 12 & 19.00 & 14.00 & 19.00 & \\
\hline & & 24 & 21.00 & 8.00 & 31.00 & \\
\hline & & 36 & 2.76 & 2.62 & 3.73 & \\
\hline & & 48 & 8.40 & 2.40 & 9.00 & \\
\hline & \multirow{5}{*}{ BSA } & 0 & 9.00 & 8.00 & 12.00 & \multirow{5}{*}{ NS } \\
\hline & & 12 & 2.48 & 1.26 & 3.01 & \\
\hline & & 24 & 6.00 & 4.80 & 7.00 & \\
\hline & & 36 & 12.00 & 11.00 & 15.00 & \\
\hline & & 48 & 11.00 & 5.00 & 14.00 & \\
\hline & \multirow{5}{*}{ DLQI } & 0 & 14.00 & 10.00 & 20.00 & \multirow{5}{*}{ NS } \\
\hline & & 12 & 16.00 & 4.00 & 28.00 & \\
\hline & & 24 & 3.44 & 2.93 & 4.95 & \\
\hline & & 36 & 4.00 & 2.00 & 6.20 & \\
\hline & & 48 & 12.00 & 8.00 & 14.00 & \\
\hline
\end{tabular}


tive. The relatively small sample group resulted from the rigorous criteria for the inclusion of patients for biologic therapy, provided for in the recommendations given by the Polish Society of Dermatology [1].

On the basis of the data concerning the evaluation of ustekinumab efficacy, it may be observed that in weeks 16 (PASI) and 28 (BSA, DLQI) of the conducted observation, there occurred an alarming increase in the used parameters of the anti-cytokine therapy efficacy. However, in the last week 40, we observed decreased values of all three indexes. Thus, it seems that the observed changes are of a temporary nature. It seems interesting that in week 28 an increased BSA value coincides with an increased DLQI value, which indicates lower comfort of patients' lives, with the concurrent drop in the PASI value, when compared with its value in week 16 . It emphasises the necessity to conduct analyses of therapy progress basing on several scales and the possible interpretation difficulties in case of each of them. The differences between the values of parameters seemed to be statistically significant, starting from week 16 of the therapy onwards.

As far as etanercept is concerned, the direction of changes in the PASI and BSA values was, generally, different during the whole observation period. Differences in the observed changes in the parameters during 48 weeks of therapy seemed to be statistically insignificant. Partially, it may result from a small group of patients treated with etanercept during the study. Basing on DLQI, the patients described that in weeks 24 and 36 of their therapy psoriasis made their functioning difficult, which is not reflected in the PASI and BSA values. Reich et al. analysed the efficacy of etanercept therapy in the group composed of 83 patients. They evaluated it by comparing the PASI values on the day of study commencement and after 16 weeks, noting clinical improvement in $48.2 \%$ of patients covered by the study [4]. In turn, our results do not confirm the downward trend in the PASI values between weeks 12 and 24 of the therapy, which is most likely correlated with the fact that the group was small.

Increased PASI and BSA values during the ustekinum$\mathrm{ab}$ and etanercept therapies may indicate resistance to the drug, which is also noted in the observations conducted by Langley et al. [5]. However, the results obtained in our study indicate that the changes are of a temporary nature. In turn, Strober et al. state that resistance to ustekinumab therapy may be determined by sex [6], which confirms the complex character of mechanisms conditioning response to treatment. They also underline greater safety and efficacy in case of ustekinumab, when compared with etanercept [6], which seems to be confirmed in our study.

In case of ustekinumab, lower PASI and BSA values were observed in week 12 of the therapy, while for etanercept not until week 24, which suggests the greater efficacy of the anti-IL12/23 medicine. Studies conducted by
Zweegers et al. also confirmed our conclusion concerning the therapeutic potential of the analysed medicines. They state that ustekinumab is more effective than etanercept. Their conclusion is based on the observation that patients treated with ustekinumab had their PASI value reduced, the therapeutic effect lasted for a year and it was not necessary to increase the dosage when compared with the group treated with etanercept [7]. Strober et al. also indicate greater efficacy of ustekinumab therapy, when compared with the therapy with the use of three TNF inhibitors (adalimumab, etanercept, and infliximab) [6].

In conclusion, the evaluation of disease intensity and the efficacy of pharmacotherapy should be made taking into account the commonly binding scales - PASI, BSA and DLQI. It must be remembered that such clinical assessment is not free of interpretation errors and difficulties. Despite the molecularly targeted anti-cytokine therapies, the unfavourable loss of adequate response to treatment is observed which occurs between week 16 and 24 of the therapy. On the basis of presented data in changed values of the said scales and review of available literature it seems that ustekinumab shows greater efficacy than etanercept.

\section{Conflict of interest}

The authors declare no conflict of interest.

\section{References}

1. Reich A, Szepietowski J, Adamski Z, et al. Rekomendacje diagnostyczno-terapeutyczne Polskiego Towarzystwa Dermatologicznego. Część II: łuszczyca umiarkowana do ciężkiej. Dermatol Rev 2018; 105: 329-57.

2. Kwiek B, Narbutt J, Sysa-Jędrzejowksa A, et al. Long-term treatment of chronic plaque psoriasis with biological drugs can control platelet activation: targeting the bridge between inflammation and atherothrombosis. Adv Dermatol Allergol 2017; 34: 131-7.

3. Bożek A, Reich A. How to reliably evaluate the severity of psoriasis? Forum Dermatol 2016; 2: 6-11.

4. Reich K, Gooderham M, Green L, et al. The efficacy and safety of apremilast, etanercept and placebo in patients with moderate-to-severe plaque psoriasis: 52-week results from a phase IIIb, randomized, placebo-controlled trial (LIBERATE). J Eur Acad Derm Venereol 2016; 31: 507-17.

5. Langley RG, Tsai TS, Flavin S. Efficacy and safety of guselkumab in patients with psoriasis who have an inadequate response to ustekinumab: results of the randomized, doubleblind, phase III NAVIGATE trial. Br I Dermatol 2018; 78: 114-23.

6. Strober BE, Bissonnette R, iorentino D, et al. Comparative effectiveness of biologic agents for the treatment of psoriasis in a real-world setting: results from a large, prospective, observational study (Psoriasis Longitudinal Assessment and Registry [PSOLAR]). J Eur Acad Derm Venereol 2016; 74: 851-61

7. Zweegers J, Groenewoud JMM, van den Reek JMPA. Comparison of the 1- and 5-year effectiveness of adalimumab, etanercept and ustekinumab in patients with psoriasis in daily clinical practice: results from the prospective BioCAPTURE registry. Br J Dermatol 2016; 176: 1001-9.. 